REPRINTED FROM

\title{
6 \\ Cognitive Learning, Cognitive Response to Persuasion, and Attitude Change ${ }^{1}$
}

\author{
ANTHONY G. GREENWALD \\ DEPARTMENT OF PSYCHOLOGY \\ OHIO STATE UNIVERSITY \\ COLUMBUS, OHIO
}

It is a common assumption that the effectiveness of a persuasive communication is, at least in part, a function of the extent to which its content is learned and retained by its audience. This assumed learning-persuasion relation is based on a reasonable analogy between the persuasive communication and an informational communication such as a classroom lecture. In the lecture, it is by definition of the educational situation that retention of content is taken as a measure of effectiveness. In the persuasion situation, however, the essential criterion of effectiveness is acceptance of content. It remains an empirical question to determine whether acceptance of a persuasive communication is related to retention of its content.

The hypothesis that acceptance of a communication is, in some part, a function of learning or retention of its content has received explicit endorsement by a number of attitude researchers and theorists (e.g., Hovland, Janis, \& Kelley, 1953; McGuire, this volume; Miller

1Preparation of this report and the research reported here were supported in large part by grants from the National Science Foundation (GS-1601) and the Mershon Social Sciences Program at Ohio State University. The author is particularly indebted to Rosita Daskal Albert, Dallas Cullen, Robert Love, and Joseph Sakumura who have participated actively in various phases of the research reported here. A condensed version of this chapter was presented at the American Psychological Association symposium, "Alternatives to consistency theory in the study of attitude change," Washington, D.C., September, 1967. 
\& Campbell, 1959; Watts \& McGuire, 1964) and has aroused no published opposition. Indeed, this cognitive learning model of persuasion is most reasonable. It is widely accepted that cognitions bearing on the object of an attitude form a major component of the structure of the attitude toward that object (see, for example, Campbell, 1947; Katz \& Stotland, 1959; Krech, Crutchfield, \& Ballachey 1962; Rokeach, 1960; Smith, Bruner, \& White, 1956). Since the individual is not born with his cognitions, but acquires them, there seems to be no reasonable alternative to the assumption that cognitions bearing on attitude objects are learned. Further, the most obvious source of such cognitions is the wealth of persuasive messages to which one is exposed via the public communications media as well as through face-to-face communications.

In light of the overpowering reasonableness of the persuasion-asa-function-of-retention hypothesis, it is rather surprising how unsupporting the research evidence is. A few studies have directly examined the relation between the learning and attitudinal effects of persuasive communications (Insko, 1964; Miller \& Campbell, 1959; Watts \& McGuire, 1964). These studies have generally found that both communication retention and persuasion diminish with increasing time between communication and posttest, consistent with the hypothesis that retention is necessary for persuasion. On the other hand, these same studies have found only weak and variable correlations between communication retention and persuasion among subjects tested at the same posttest interval, suggesting that the relation between retention and persuasion is not a necessary one. In a conceptually relevant study on impression formation, Anderson and Hubert (1963) have concluded that there are separate memory systems for retention of a set of person-descriptive adjectives and of the person-impression derived from them; their results thus also suggest little or no necessary relation between a communication's retention and its effectiveness. Additionally, in a number of studies scattered throughout the attitude literature (and reviewed in Hovland et al., 1953), variables shown to affect opinion demonstrably - such as credibility, fear arousal, and organization of arguments - have not been found to have corresponding effects on retention of communicated arguments. Such negative findings on retention measures have typically been used to counter any possible interpretation for obtained attitude change differences in terms of unintentionally induced differences in attention to or retention of communication content In sum, the research evidence must be interpreted as uncongenial to the hypothesis that persuasion is a function of retention of per- suasive arguments. (In partial reviews of the relevant literature, Insko, 1967, and McGuire, 1968, reach similar conclusions.)

It must be concluded that either (a) learning of attitude-relevant cognitions (i.e., persuasive arguments) is unrelated to attitude formation and change; or (b) persuasive communications can induce attitude change without necessarily providing the cognitive content on which the attitude is based. The first conclusion carries the implication that learned cognitions are not fundamental to the structure of attitudes. Rather than accept this conclusion, which runs counter to most conceptions of attitude, it seemed worth some effort to explore sources other than persuasive communications as possible origins of learned attitudinal cognitions.

\section{COGNITIVE RESPONSES TO PERSUASION}

There is, of course, an important extracommunication source of cognitive content in the persuasion situation: the cognitive reactions of the communication recipient to incoming persuasive information. When a person receives a communication and is faced with the decision of accepting or rejecting the persuasion, he may be expected to attempt to relate the new information to his existing attitudes, knowledge, feelings, etc. In the course of doing this, he likely rehearses substantial cognitive content beyond that of the persuasive message itself. The present hypothesis is, then, that rehearsal and learning of cognitive responses to persuasion may provide a basis for explaining persisting effects of communications in terms of cognitive learning. The learning of cognitive response content may, indeed, be more fundamental to persuasion than is the learning of communication content.

This hypothesis is not a new one. The following passages from previous works indicate views bearing the essence of the present hypothesis.

. there is reason to expect that those audience members who are already opposed to the point of view being presented may be distracted [from the content of a communication] by "rehearsing" their own arguments while the topic is being presented and will be antagonized by the omission of the arguments on their side [Hovland, Lumsdaine, \& Sheffield, 1949, p. 201].

When exposed to [a persuasive communication], a member of the audience is assumed to react with at least two distinct responses. He thinks of his own [opinion], and also of the [opinion] suggested by the communicator. Merely thinking about the new opinion along with the old would not, in itself 
lead to opinion change. The individual could memorize the content of the [new opinion] while his opinion remained unchanged. Practice, which is so important for memorizing verbal material in educational or training situations, is not sufficient for bringing about the acceptance of a new opinion [Hovland et al., 1953, p. 11].

It was hypothesized that conformity in the communication situation will increase attitude change to the extent to which implicit supporting responses are produced, and decrease attitude change to the extent to which implici interfering responses are produced...

By supporting response is meant any implicit response made by the individual (usually a self-verbalization), which provides arguments in favor of the overt response he makes; which produces further motivations in the direction of the overt response; or which relates the overt response to other stimulus situations. By interfering response is meant any implicit response made by the individual which provides motivation against the overt response he makes; which limits the stimulus situations to which the overt response is applicable; or which is generally irrelevant (such as aggressive or distracting responses) [Kelman, 1953, p. 187, 211].

Despite a number of speculations similar to the ones just cited, there has been no direct experimental exploration of the role of cognitive responses in persuasion, and, in fact, there is not much research that is even relevant. The research on active participation in the communication process (e.g., Hovland et al., 1953, Ch. 7) comes closest to being relevant. Elsewhere, isolated experiments (e.g., Brock, 1967; Janis \& Terwilliger, 1962; Kelman, 1953) have explored dependent variables approximating the present conception of cognitive responses to persuasion. Research in which cognitive responses to persuasion are employed as independent variables in experimental persuasion situations is particularly needed. Before proceeding to a consideration of evidence collected in the author's laboratory, it will be useful to state the present hypothesis with some precision.

It is proposed that the persuasion situation is usefully regarded as a complex stimulus that evokes in the recipient a complex cognitive response. The essential dimensions of the recipient's cognitive response are, at the least, (a) response content, i.e., degree of acceptance versus rejection of the position advocated in the communication, and (b) intensity, or vigor, of response. The latter dimension, as well as other possible dimensions of cognitive response, will not be considered further in this chapter. The essential components of the persuasion situation as a stimulus - that is, as determinant of the cognitive response content-are setting, source, and communication content. An additional major set of determinants of the cognitive response content is the set of characteristics brought by the recipient to the persuasion situation, including his existing repertory of attitude-relevant cognitions as well as personality traits and group memberships.

As in many other treatments of persuasion, the cognitive response analysis assumes that attitude change can be achieved by the modification, through learning, of the recipient's repertory of attituderelevant cognitions. Such modification might include strengthening of existing cognitions as well as introduction of new ones. The present emphasis on the mediating role of the recipient's own cognitive responses to persuasion may be formulated as an assertion that cognitive modification of attitudes requires active (not necessarily overt) rehearsal of attitude-relevant cognitions at a time when the attitude object or opinion issue is salient. Thus the effects of persuasive communications might range from persuasion - when the recipient rehearses content supporting the advocated position-to boomerang-when the recipient rehearses content opposing the advocated position.

As a consequence of the present emphasis on the recipient's rehearsal of his own responses to persuasion, it is assumed that learning of communication content does not play an essential role in mediating the effects of persuasive communications. The present formulation, therefore, is capable of maintaining an analysis of persuasion effects in terms of cognitive learning while being compatible with findings indicating no necessary relation between communication retention and persuasion.

It is possible to formulate the cognitive response analysis in terms of an analogy to the classical conditioning paradigm. In this analogy, the persuasion situation corresponds to the unconditioned stimulus in that it has a response evocation capacity; that is, it influences the content of the recipient's cognitive response. As an analog of the unconditioned response, the cognitive response becomes transferred to the attitude object, which is analogous to the conditioned stimulus of the classical paradigm. While this analogy may be decidedly useful, especially in relating the present analysis to other treatments that have invoked the classical conditioning model (see the chapters by Staats and Weiss in this volume), it would be inappropriate currently to regard the model as more than a possibly suggestive analogy. A point of difficulty that would arise if the model is taken literally, for example, would concern the nature of the conditioned response in persuasion; it would be unnecessarily cumbersome, at this stage, to incorporate in the present analysis an analogy to the conditioning model's assumption that conditioned responses are either fractional 
components of unconditioned responses or preparatory adjustments to unconditioned stimuli.

\section{STUDIES OF COGNITIVE LEARNING AND ATTITUDE \\ CHANGE}

The cognitive response analysis of persuasion has guided a series of experiments conducted at Ohio State University. While the pri mary focus of this research has concerned the properties and functions of recipients' cognitive responses in persuasion situations, a secondary focus has been on the role of communication content in persuasion. The present section is devoted largely to the findings of completed portions of this research program. The first two subsections deal with experiments in which communication content learning and cognitive response rehearsal have been employed as manipulated independent variables, enabling conclusions about their causal involvement in persuasion. The next two subsections are concerned with experiments in which retention of communicated arguments and occurrence and retention of cognitive responses have been observed as dependent variables; from these experiments it is possible to draw conclusions about the determinants and correlates of cognitive response content and of retention of cognitive responses and communication content. A final subsection provides a brief review of literature on variables influencing the retention of communicated persuasive arguments.

It is to be emphasized that the research program described here is very much in progress. At appropriate points below, the nature of planned and in-progress research will be indicated; these comments will serve, perhaps, more to indicate the author's awareness of questions that remain to be answered than to enlighten the reader

\section{AtTitude Change and Learning of Communication Content}

Previous studies of the relationship between communication retention and opinion change have tested this relationship correlationally - subjects received a communication and were subsequently tested for both retention of content and acceptance of the viewpoint of the communication. With the assistance of Rosita Albert and Dallas Cullen, the author conducted a study (unpublished) in which communication retention was a manipulated independent variable, with opinion as the dependent variable. Such a design, it may be noted is more appropriate to drawing a conclusion about the causal role of communication learning in attitude change than is a correlational design.

The experiment employed three communications concerning United States foreign aid policy. One of these consisted of 6 brief arguments favoring foreign aid, 1 of 6 arguments opposing foreign aid, while the third was composed of all 12 of these arguments and did not draw either a favorable or unfavorable conclusion. For each communication, a group of subjects was asked to rehearse the individual arguments contained in it, in preparation for a subsequent retention test; in addition to these three groups, two other groups received the 12 -argument communication, and, after briefly reading it once, in entirety, were asked to rehearse only 6 arguments that had been underlined. For one of these groups, the 6 underlined arguments were those favoring foreign aid, while for the other group the 6 opposing foreign aid were underlined. Subjects were tested for opinion on the issue and then for retention of all arguments in their communications (not just rehearsed ones) immediately following the learning task and again, unexpectedly, after a 1-week interval.

For the group receiving the one-sided pro-foreign aid message $(N=16)$, an average of 3.7 (out of 6) arguments were retained for the immediate retention test and 3.1 for the unexpected retention test one week later. The corresponding means for the group receiving the one-sided anti-message $(N=20)$ were 3.6 arguments retained immediately and 2.4 after a week. Since these two groups differed significantly from each other in opinion in the directions advocated in their communications, both immediately $(F=9.50, d f=1,34, p<.01)$ and after a week $(F=7.03, p<.02)$, these data were consistent with the hypothesis that communication learning and persuasion are related. Among subjects receiving the two-sided communications, those assigned to learn pro arguments $(N=18)$ retained substantially more pro than con arguments $(p<.001$, for each testing); the reverse was true, as expected, for those $(N=19)$ asked to rehearse con arguments ( $p<.001$, for each testing); and those asked to learn both sets $(N=18)$ retained approximately equal numbers of each.

The effectiveness of the partial learning assignments for the two-sided communications can be indicated by the fact that subjects retained an average of 3.0 assigned arguments compared to 0.3 nonassigned ones on the immediate retention test; for the delayed retention test, the corresponding means were 1.8 assigned and 0.3 nonassigned arguments. If there is, indeed, a causal relation between argument learning and persuasion, these manipulated differences in retention should have produced corresponding differences in opinion. 
However, there were no opinion differences among the three groups receiving two-sided communications, either immediately $(F<1$, $d f=2,52)$ or after a week $(F<1)$.

It must be concluded that learning of communication content is not a sufficient condition, and perhaps not even a necessary condition, for persuasion. That is, learned arguments supporting one side of the foreign aid issue were ineffective when, at the time of learning, subjects were made aware that credible opposing arguments were available-even though the opposing arguments were poorly retained. This finding stands in contrast with those of studies demonstrating across-cell correlations between learning and persuasion (e.g., Insko, 1964; Miller \& Campbell, 1959; Watts \& McGuire, 1964). The lack of opinion differences between conditions with decided retention differences in the present study suggests that such previously obtained across-cell correlations should not be interpreted in terms of a causal relation between communication retention and persuasion.

\section{ACCEPTANCE AND RECALL OF IMPROVISED ARGUMENTS}

If, as is presently supposed, attitudes change in the direction of cognitive content rehearsed during a persuasion situation, then procedures that manipulate the content of the recipient's cognitive responses should have persuasive effect. A traditional persuasion procedure that may be viewed as a manipulation of cognitive response content is the improvised role-playing procedure in which a subject is asked to deliver a persuasive message supporting a position initially unacceptable to him. The majority of research evidence indicates that role playing produces greater persuasion toward the unacceptable position than does passive receipt of a persuasive communication (cf. Insko, 1967, p. 222). Such results are quite compatible with the present point that a communication recipient's rehearsal of his own arguments may be more important in persuasion than is his rehearsal of arguments contained in a communication to which he is exposed. However, some commentators feel that the currently available evidence on role playing is equivocal (see McGuire, 1966, p. 498). Because of this empirical uncertainty, the present research program included an experiment intended to assess the effect of improvised role playing on both acceptance and retention of arguments.

In this experiment (Greenwald \& Albert, 1968), each subject improvised five arguments in response to an assignment to advocate either specialized (career preparatory) or general (liberal arts) under- graduate education. Improvisation was obtained in response to five neutrally worded questions that could be answered with an argument supporting either viewpoint. Assignment to positions was random with approximately 90 subjects being assigned to each side. In addition to being exposed to their own improvised arguments supporting one side of the issue, subjects carefully read and studied-for about the same amount of time they had spent improvising-a set of arguments supporting the opposite side that was actually written by another subject in the study. Since each subject's improvisations served once as an improvised set of arguments and once as an externally originated set (for a different subject), this procedure served to equate quality of arguments for the two sets over the sample of subjects, although not necessarily for each subject. After a 20 -minute irrelevant task, the subjects were tested for opinion on the generalspecialized education issue and were then asked to recall as many arguments as they could of those to which they had been exposedboth their own and the ones that had been improvised by another subject.

Figure 1 gives the opinion and retention results for this study. It may be seen that subjects arrived at opinions consistent, on the average, with the position to which they had been assigned. The opinion difference between the two groups was statistically significant $(F=7.42, d f=1,177, p<.01)$. A more powerful effect was obtained with the retention measure, with subjects tending to recall much more of their own improvised arguments than they did of those improvised by another subject $(F=168.10, d f=1,777, p<.001)$. A supplementary finding was that subjects tended to rate their own arguments as more original than the other's $(p<.001)$, indicating that, other things being equal, one tends to evaluate his own thoughts more favorably than others'. In summary, these results demonstrated that the subject's rehearsal of his own cognitions and externally originated cognitions of comparable quality tended to result in attitude change in the direction of the content of the subject's own cognitions. These results support the general trend of findings in the role-playing literature. More importantly for present purposes, they add force to the hypothesis that the recipient's rehearsal of his own cognitive responses plays an important role in persuasion.

It should be noted that the Greenwald-Albert experiment does not provide an explanation of the special efficacy of the recipient's own improvised arguments. It could be that subjects' increased retention of their own arguments was responsible for the observed attitude effect; however, alternative explanations are possible in terms of 


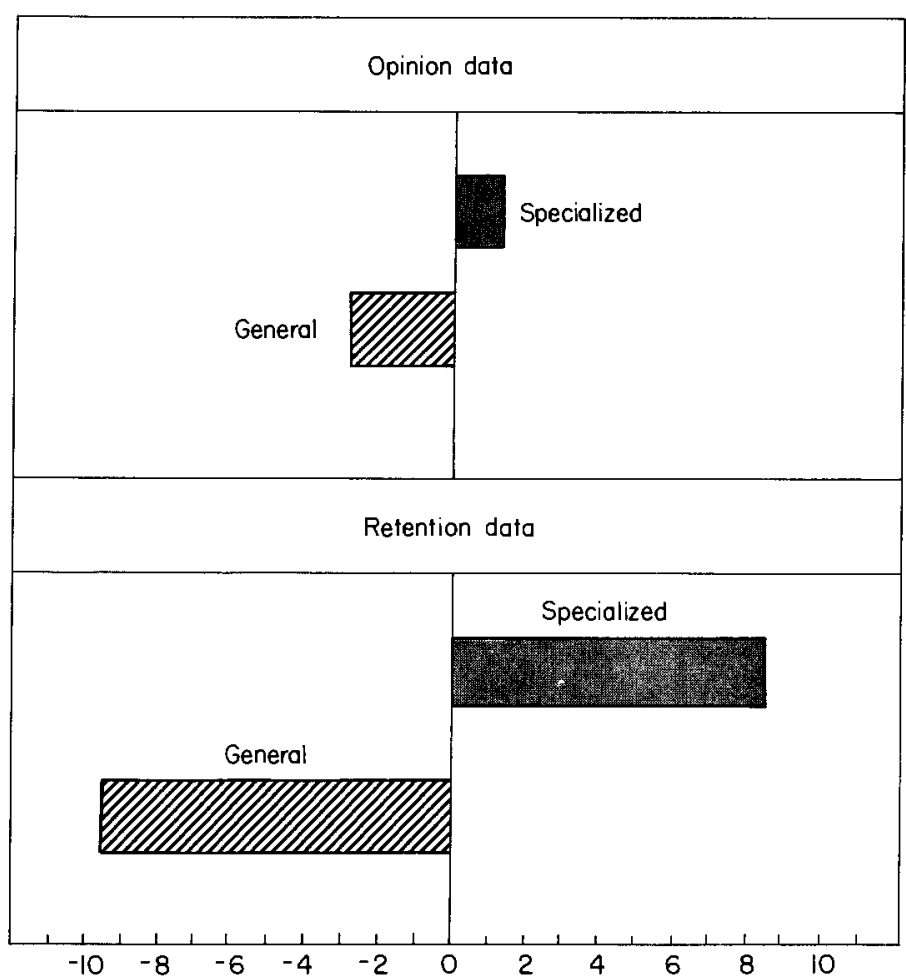

SD units for mean of 90 subjects

FIG. 1. Opinion and retention as a function of position assigned for improvisation. (Bars are labeled with the assigned position. Positive scores on the opinion measure represent favorableness to specialized education; positive scores on the retention measure represent greater recall of arguments favoring specialized education than of ones favoring general education.) (Greenwald \& Albert, 1968.)

perception of the self as the source of arguments or in terms of subjects' ability to generate arguments particularly effective in regard to their own attitude structure (cf. Hovland et al., 1953, pp. 233-237). A series of experiments designed to select among these explanations will be conducted in the near future as part of the present research program.

\section{CONTENT and CORRelates of Cognitive Responses to} Persuasion

A series of experiments conducted by Dallas Cullen, under the author's supervision, explored the content of subjects' thoughts relevant to the topic of a persuasive communication that they had just received. The communication, on the issue of general versus specialized undergraduate education, advocated the view that college education should be directed specifically at career preparation (that is, should be specialized). It was assumed that cognitive effects of the communication could be assessed by comparing the issue-relevant thoughts of subjects who received this communication with thoughts on the same issue expressed by control subjects who had received a communication on a different topic.

The subjects in this series of experiments were Ohio State University introductory psychology students who participated in classroom size groups. Four separate experiments were conducted with only minor variations in procedure. The basic procedure for Experiment I included an introduction to the issue of general versus specialized education consisting mainly of a definition of the alternative positions. Then a communication of about 250 words containing 12 distinct arguments supporting specialized undergraduate education was administered in printed form. Control subjects, at this time, read a communication concerning instructional television. Next, all subjects (including controls) were asked to "collect their thoughts" on the issue of general versus specialized undergraduate education by listing thoughts that were:

... pertinent to forming and expressing an opinion on the issue of general versus specialized education. These thoughts may consist of (a) information favorable to one or the other viewpoint; (b) personal values of yours that are favorable to one or the other viewpoint; (c) features of either viewpoint that you perceive as good; (d) features of either viewpoint that you perceive as bad or harmful; and (e) any other thoughts you feel to be pertinent.

In writing down these thoughts, please separate your thoughts into individual ideas to be written down separately. An "individual idea" is one that, to the best of your judgment, expresses only a single fact, value, good or bad feature, or thought.

Following this "thought-listing" procedure, subjects completed a brief questionnaire of four Likert-type items measuring opinion on the general-specialized education issue. Finally, subjects were instructed to look back at the thoughts they had listed concerning general and specialized education and to judge, for each individual thought, whether it was favorable to general or specialized education and how favorable it was, on a three-point scale of slightly favorable (1), moderately favorable (2), and very favorable (3).

Experiment II added an opinion pretest to the basic procedure; it 
consisted of the four Likert-type items also used as the opinion posttest.

For Experiment III, subjects were given an additional judgment task, at the end of the experiment, in regard to their listed thoughts. They were to assign each thought to one of three categories: (a) those having their source in the experimental materials (the introductory definitions and the persuasive communication); (b) modifications of the experimental materials (such as illustrations of, qualifications of, and reactions to communicated arguments); and (c) ideas not traceable to the experimental materials. These three categories are to be identified here, respectively, as (a) externally originated, (b) recipient-modified, and (c) recipient-generated cognitions. The aim of Experiment III was to compare subject coding of listed-thought responses into these three categories with independent coding by judges; no control group was employed.

In the last experiment in this series, two different communication conditions were employed. One (group IVA) employed the same communication used in the previous experiments while the other (group IVB) was modified by adding material acknowledging opposing arguments (ones favoring general education) and refuting them where possible (cf. Hovland et al., 1949, Ch. 8). Subjects in Experiment IV also performed the additional categorization of their listed thoughts that had been requested of subjects in Experiment III.

For Experiments I, II, and III the classification of thoughts into externally originated, recipient-modified, and recipient-generated categories was performed by judges who were able to agree on these classifications for $85 \%$ of their judgments. This extent of agreement was not considered entirely satisfactory, especially when it was found, in Experiment III, that judges' classifications agreed with subjects' classifications for only $62 \%$ of judgments (chance agreement would be $33 \%$ ). Since subjects were able to perform the judging task with relative ease, it was decided that it would be most satisfactory to use subject self-scoring for subsequent groups.

The data for the four experiments were first examined in terms of the quantities of thoughts as distributed among the three categories externally originated, recipient-modified, and recipient-generated. These data are given in Fig. 2. Judges' categorizations were used for Experiments I and II; for Experiments III and IV, subjects' own categorizations were used. The most significant feature of the data summarized in Fig. 2 is that the recipient-generated category accounted for the majority of thoughts listed by communicationreceiving subjects-despite the fact that their thoughts were tapped
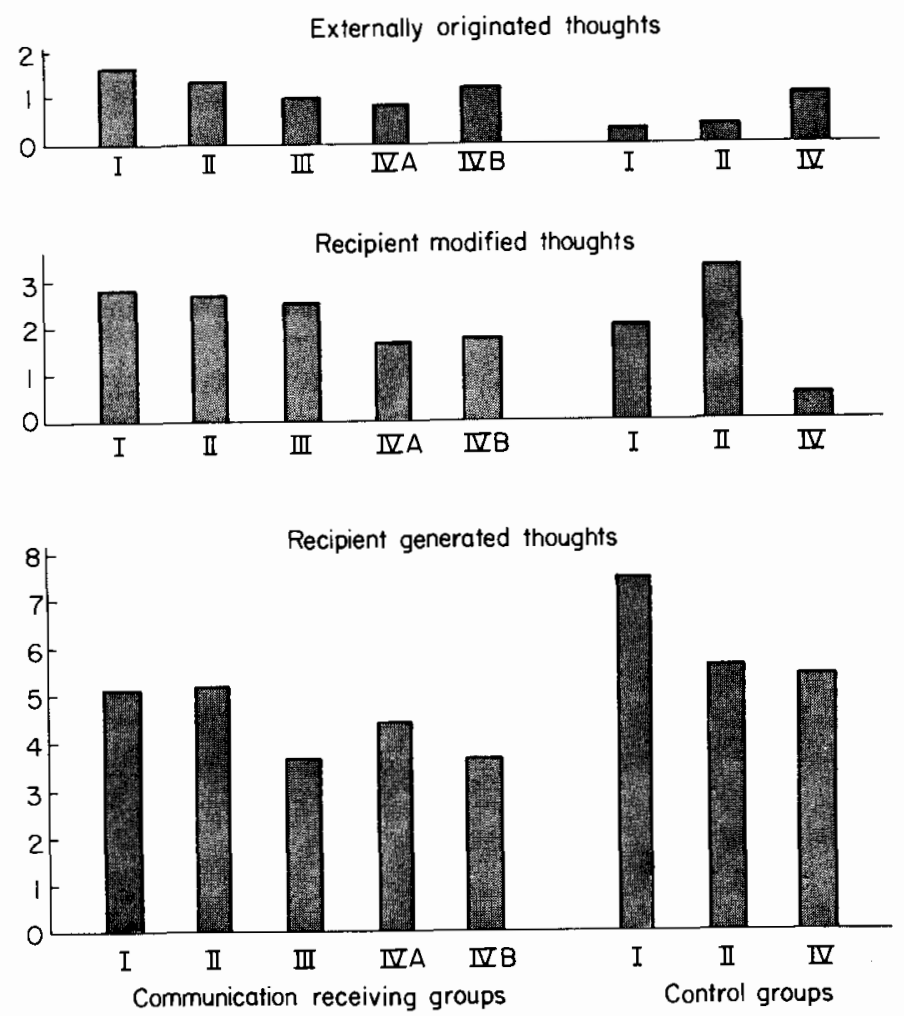

FIG. 2. Mean quantities of listed thoughts in three categories. (Numbers of subjects are as follows: communication-receiving groups: I (48), II (48), III (68), IVA (26), IVB (28); control groups: I (16), II (16), IV (13).) (Greenwald \& Cullen, unpublished data.)

immediately after reading a communication containing a dozen distinct relevant thoughts that could have been listed. ${ }^{2}$

Subjects' judgments of the position supported by each of their listed thoughts were used to calculate an index summarizing the thoughts' content on the general-specialized education issue. It will be recalled that each thought was weighted according to its degree of support for the position it supported. The index was calculated by subtracting the sum of weights for thoughts favoring general education

${ }^{2}$ The quantities of listed thoughts in the externally originated and recipient-modified categories for control groups were greater than zero because control subjects could and did list or react to material contained in introductory definitions of the concepts of general and specialized education. 


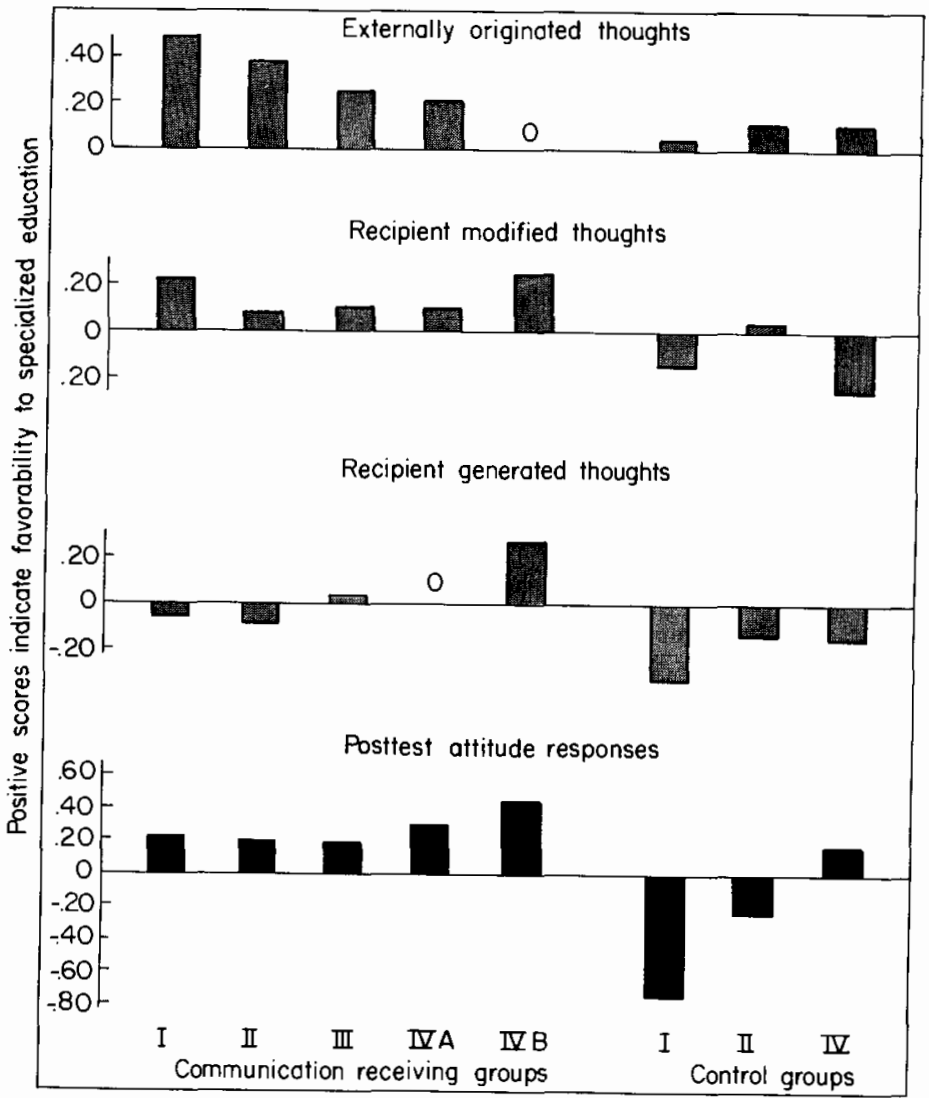

Fic. 3. Mean directional content of listed thoughts in three categories and mean posttest attitude responses. (The extreme scores on the three category indexes are +1.00 and -1.00 ; for the posttest attitude responses, the extremes are +2.50 and -2.50.) (Greenwald \& Cullen, unpublished data.)

from the sum for those favoring specialized education, then dividing this difference by the sum of weights for all thoughts. This calculation was done separately for thoughts in each of the three categories - externally originated, recipient-modified, and recipient-generated. Figure 3 gives the mean values of these directional content indexes for the various groups in the present series of experiments. For comparison, the mean posttest opinion questionnaire responses are given at the bottom of Fig. 3 . The effects of the communications can be seen in the generally more positive index values and posttest opinion scores for communication groups than for controls. The significance levels for these comparisons, combining over Experiments I, II, and IV (no control group was used in Experiment III) were: externally originated category, $p<.02$; recipient-modified category, $p<.02$; recipient-generated category, $p<.07$; and posttest opinion, $p<.001$ (all one-tailed).

It may be noted that the communication received by subjects in group IVB - which differed from the others in that it acknowledged opposing arguments - produced the most favorable posttest opinion questionnaire responses and the most positive responses in the recipient-generated category, while producing the least positive responses in the externally originated category. This pattern of findings is suggestive of the importance of recipient-generated cognitions, relative to externally originated ones, in persuasion.

Figure 4 gives for each group the correlations with posttest opinion for the three category directional content indexes as well as for one based on all thoughts combined. It may be noted that the correlations involving the recipient-generated category index were quite high relative to those for the externally originated category. This suggests, once more, the importance of recipient-generated cognitions in the recipient's attitude structure. ${ }^{3}$

The correlations with posttest opinion for the recipient-generated category index and for the index based on all thoughts combined were sufficiently high to suggest that the thought-listing procedure used in the present experiments might be very useful as a measure of opinion. A very desirable aspect of the thought-listing procedure is that it is applicable to virtually any attitude issue without necessity for time consuming scaling and item selection procedures. Research currently underway, being conducted by Cullen, is exploring the reliability, validity, and sensitivity-to-change properties of the thought-listing procedure in comparison with more traditional attitude scaling procedures. (See experiment reported on pp. 163-165 for an illustration of the use of the thought-listing procedure as an opinion measure.)

Table 1 gives the additional correlational data that were obtained

${ }^{3}$ Split-half reliability of the externally originated category index (.74) was actually slightly higher than that for the recipient-generated category (.68), which indicates that the difference between correlation magnitudes could not be attributed to relative unreliability of the externally originated category index. However, it must be noted that reliability of the externally originated category index. However, it must be noted that
the externally originated material was nearly uniformly favorable to the specialized education position; thus, it is possible that range restriction for the externally originated category index was responsible, in part, for the low-magnitude correlations obtained between externally originated thought content and posttest opinion. 


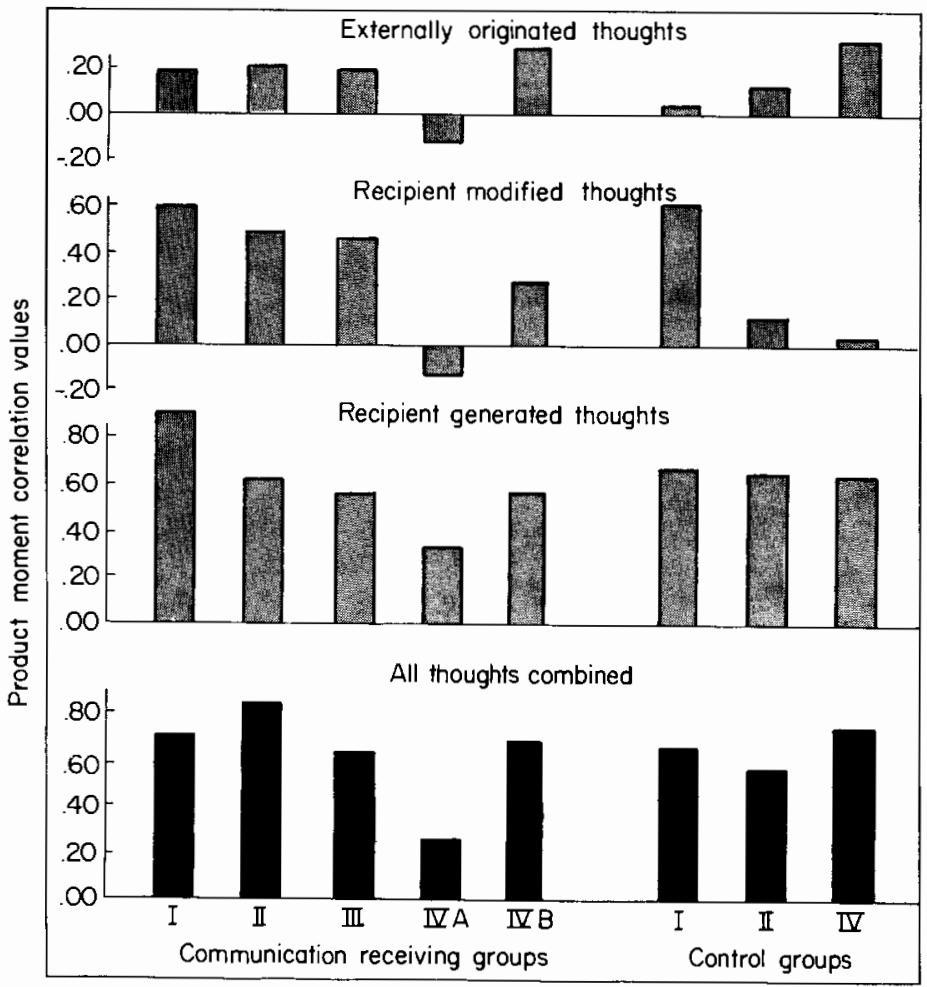

FIG. 4. Correlations with posttest attitude for four listed thought directional content indexes. (Greenwald \& Cullen, unpublished data.)

TABLE 1

Correlations of Listed Thought CONTENT Indexes with Pretest and Posttest AtTitude Measures ${ }^{\prime \prime}$

\begin{tabular}{lcc}
\hline & \multicolumn{2}{c}{ Correlations with } \\
\cline { 2 - 3 } \multicolumn{1}{c}{ Content index } & Pretest attitude & Posttest attitude \\
\hline Externally originated thoughts & .20 & .06 \\
Recipient-modified thoughts & $.56^{c}$ & -.02 \\
Recipient-generated thoughts & $.52^{c}$ & $.39^{c}$ \\
All thoughts combined & $.7 \mathrm{l}^{c}$ & $.65^{c}$ \\
\hline
\end{tabular}

${ }^{a}$ Experiment II Data; $N=48$.

${ }^{b}$ Pretest altitude has been partialed out of correlations with posttest attitude. ${ }^{c} p<.01$. from Experiment II by virtue of the use of a pretest opinion measure. On the left side of the table, it may be seen that pretest opinion was positively correlated with all of the directional content indexes, indicating that pretest opinion was an important determinant of the content of cognitions rehearsed in response to the persuasion situation. On the right side of the table, correlations between the category directional content indexes and posttest opinion are given, with pretest opinion partialed out. Significant positive correlations were obtained only for the recipient-generated category index and for the one based on all thoughts combined-again suggesting the importance of recipient-generated cognitions in persuasion, relative to externally originated ones.

In summary, Cullen's experiments provided a variety of evidence indicating important involvement of cognitive responses to persuasion in attitude structure. These findings, especially those for recipient-generated cognitive responses, were consistent with the present hypothesis that rehearsal of cognitive responses to persuasive communications is an essential mediator of cognitive attitude change.

\section{Persuasion and Retention of Cognitive Responses}

The preceding series of experiments examined attitude-relevant cognitions that were recorded shortly after the receipt of a persuasive communication. Robert Love's master's thesis research (in preparation), conducted under the author's direction, examined recipient's cognitions during a persuasive communication situation. The aim of this experiment was chiefly to assess correlations of persuasion effectiveness with retention of communication content and retention of cognitive response content, observed immediately following and one week after the initial communication situation.

Love's sample was divided into two groups, one receiving a persuasive communication on admitting Puerto Rico as the fifty-first state $(N=33)$, the other receiving a communication advocating popular election of the Secretary of State $(N=35)$; both communications were adapted with minor modifications from ones used by Watts and McGuire (1964). Subjects first received an opinion pretest (Likerttype), following which the appropriate communication was administered in printed form. Each communication presented three main supporting arguments in separate paragraphs: following each paragraph, four blank lines were provided for the subject to write a onesentence reaction to the main point of the paragraph (the main point 
was underlined in the printed communication). This procedure served to obtain a sample of cognitive responses rehearsed during the persuasion situation. ${ }^{4}$ After the communication, subjects' opinions were again assessed, this time using the thought-listing procedure (see p. 157), following which subjects were given an unexpected test for recall of (a) the three main points of the communication and (b) the reactions that had been written following each. The same subjects were recruited for an ostensibly different experiment, one week later, at which time the opinion posttest and the recall test were unexpectedly readministered.

The data were analyzed for partial correlations (pretest partialed out) of posttest opinion with (a) the content of cognitive reactions to the communication (number of favorable minus number of unfavorable reactions), (b) retention of the main arguments of the communication (number of arguments recalled; maximum $=3$ ), and (c) retention of cognitive reactions (number of favorable minus number of unfavorable reactions recalled). These correlational results are given in Table 2. It is apparent that the best predictor of the effect of the communications was the measure of content of the cognitive reactions that were written during the communication exposure (average of $4 r$ 's $=.52$ ); the next best predictor was the retention index for the cognitive reactions (average of $4 r^{\prime} s=30$ ); decidedly the poorest predictor was the retention measure for the persuasive communication itself (average of $4 r^{\prime} s=.03$ ). These results strongly support the present contention that cognitive responses to persuasion are important in mediating persuasion effects while retention of communication content is not.

Supplementary data were obtained from subjects' classifications of the thoughts they had listed for the opinion posttests into the categories of (a) having originated in the persuasive message, (b) having originated in written reactions to the persuasive message, and (c) traceable to neither of these sources. Subjects' written reactions were most often represented in their listed thoughts (mean number $=2.26$ ),

${ }^{4}$ Note that this procedure explicitly encouraged rehearsal of recipients' cognitive responses in that subjects were instructed to produce thoughts and to spend time writing their thoughts. This removes the present experimental situation a bit from the type of communication situation involving only a one-way transmission from source to recipient However, this was unavoidable in the interests of obtaining usable information about the content of recipients' cognitions during persuasion. Moreover, the situation was analogous to another important type of persuasion situation in which source and recipient are in face-to-face confrontation, the source typically being interrupted by the recipient's reactions to the communication.
TABLE 2

Partial Correlations of Cognitive Reaction and Retention Measures With IMMediate and Delayed POSTTEST OPINION

\begin{tabular}{lcccc}
\hline & \multicolumn{4}{c}{ Communication topic } \\
\cline { 2 - 5 } & \multicolumn{2}{c}{$\begin{array}{c}\text { Puerto Rico } \\
(N=33)\end{array}$} & $\begin{array}{c}\text { Secretary of State } \\
(N=35)\end{array}$ \\
\cline { 2 - 5 } & $\begin{array}{c}\text { Immediate } \\
\text { opinion } \\
\text { posttest }\end{array}$ & $\begin{array}{c}\text { Delayed } \\
\text { opinion } \\
\text { posttest }\end{array}$ & $\begin{array}{c}\text { Immediate } \\
\text { opinion } \\
\text { posttest }\end{array}$ & $\begin{array}{c}\text { Delayed } \\
\text { opinion } \\
\text { posttest }\end{array}$ \\
\hline Predictors of posttest opinion & $.47^{a}$ & $.52^{a}$ & $.59^{a}$ & $.48^{a}$ \\
$\begin{array}{l}\text { 1. Content of cognitive reactions } \\
\text { during communication }\end{array}$ & -.12 & - & .19 & - \\
$\begin{array}{c}\text { 2. Retention of communication content } \\
\text { Immediate }\end{array}$ & - & .15 & - & -.09 \\
$\begin{array}{c}\text { Delayed } \\
\text { 3. Retention of cognitive reaction } \\
\text { content } \\
\quad \begin{array}{l}\text { Immediate } \\
\text { Delayed }\end{array}\end{array}$ & .22 & - & .23 & - \\
\hline
\end{tabular}

Note: These data are from a master's thesis by Robert E. Love (in preparation). Pretest opinion was partialed out of the correlations reported in this table.

${ }^{a} \mathrm{p}<.01$, one-tailed.

${ }_{\mathrm{b}}<.05$, one-tailed

while the communication content was least often represented (mean number $=1.21$ ). (An average of 1.90 thoughts was traceable to neither source.) These findings lend further support to the conclusion that cognitive responses to the communications were more significant in providing content for cognitive attitude change than were the communications themselves.

\section{The Cognitive Learning Process in Persuasion}

The chief theoretical aim of this chapter has been to establish the basis for useful analysis of attitude change as a cognitive learning process. To do this, it was necessary to focus on cognitive responses rehearsed during persuasion situations. Specific learning-theoretical topics, such as the roles of incentives, reinforcers, and conditions of practice, have been ignored for the moment.

At present, knowledge concerning the determinants of learning of attitude-relevant cognitions is quite limited. Certainly, much is known theoretically about verbal learning, including learning of 
meaningful material (see McGeoch \& Irion, 1952; Woodworth \& Schlosberg, 1954). However, the particular variables involved in attitude-relevant learning - for example, covert rehearsal, preexisting attitudes, prior familiarity with information, and comprehension of persuasive messages - are not well understood in learning-theoretical terms. Number of presentations of a persuasive argument is about the only variable that is unequivocally established as a determinant of argument retention (see, for example, Greenwald \& Sakumura, 1968; Jones \& Kohler, 1958; Levine \& Murphy, 1943; Waly \& Cook, 1966). Until quite recently, another widely accepted principle of learning of persuasive arguments was that audiences would selectively attend to and remember information consonant with their pre-existing attitudes. Several studies had demonstrated effects of this nature (e.g., Jones \& Kohler, 1958; Levine \& Murphy, 1943). Recent attempts to replicate this phenomenon (Greenwald \& Sakumura, 1968; Waly \& Cook, 1966) have met with absolutely no success, so that the phenomenon of selective learning of attitudeconsonant information must currently be regarded as of dubious validity.

Other determinants of attitude-relevant learning that have been implicated by empirical research are information utility (Jones \& Aneshansel, 1956) and novelty (Greenwald \& Sakumura, 1968). Since utility and novelty are variables known to increase attention to persuasive information, ${ }^{5}$ it appears likely that their effects on learning of persuasive arguments may be mediated by these attentional effects rather than by any direct role in the learning process.

The findings of the studies just mentioned, and of other relevant studies not cited here, do not require the supposition that any variable other than duration of exposure (i.e., attention) to persuasive information is a determinant of information learning (cf. Cooper \& Pantle, 1967). It seems likely also that comprehension of information is a determinant of retention (see Fitzgerald \& Ausubel, 1963); however, minimal evidence is available concerning this relationship. Additionally, conditions of practice, particularly distribution of practice over time, should be expected to affect learning of persuasive information in much the same manner that they affect learning of other verbal material; again, little pertinent evidence is available, although the literature concerned with primacy and recency effects in persuasion (see Rosnow's chapter in this volume, and references;

${ }^{5}$ Brock, T. C., Albert, S. M., \& Becker, L. A. Familiarity, utility and supportiveness as determinants of information receptivity. (in preparation) also Anderson \& Hubert, 1963) may be interpreted in terms of the conditions-of-practice variable.

The effects of rewards and punishments occurring in the persuasion situation are certainly relevant to theoretical interpretations of cognitive learning. However, although effects of rewards and punishments on attitude measures have frequently been demonstrated, the processes underlying such effects are poorly understood. Competing explanations in terms of classical conditioning, instrumental learning, dissonance reduction (Brehm \& Cohen, 1962), and attention mechanisms (Janis \& Gilmore, 1965), all can be justified by appeal to portions of the relevant literature. (The reader will find extensive discussion of reward and punishment effects, interpreted in terms of conditioning processes, in the chapters by Lott and Lott, Rosnow, Staats, and Weiss in this volume.)

The procedure of having subjects actively rehearse their own persuasive arguments was found by Greenwald and Albert (1968; also summarized earlier in this chapter) to produce substantial enhancement of argument retention as well as noticeable self-persuasion. At the moment, it is unknown whether these effects were due to enhanced original attention to the improvised arguments or to other factors. Nonetheless, the focus on learning of persuasive arguments actively rehearsed in a persuasion situation has provided the basis for presently reasserting the importance of cognitive learning in persuasion. Thus, the study of determinants of persuasive-argument learning-a problem area in which, to summarize the present brief literature review, current ignorance is considerable - can be justified not only as an interesting exercise in learning theory, but in terms of its practical value in interpreting the basis for effective and durable persuasion.

\section{CONCLUSION-COGNITIVE PROCESS, COGNITIVE LEARNING, AND ATTITUDE CHANGE}

The present program of research set out to establish the legitimacy of a conception of attitude change through persuasive communication as, at least in part, a cognitive learning process. In the course of doing this, the obtained experimental evidence repeatedly indicated that the effects of persuasive communications are strongly mediated by the content of attitude-relevant cognitions elicited (and thus rehearsed and learned) during the persuasion situation. The analysis of determinants of cognitive response content may very well require 
explanatory principles outside the scope of the learning principles with which the present research was concerned. There is, however, no lack of theories in the cognitive integration area that might be applied to the analysis of determinants of cognitive response content. Cognitive consistency theories, for example, can be used to predict that cognitive responses to persuasion will be consistent with preexisting attitude-relevant cognitions. The assimilation-contrast approach (Sherif \& Hovland, 1961) predicts that the individual reacts with favorable cognitions to persuasive statements within his latitude of acceptance and with unfavorable cognitions to statements outside his latitude of acceptance. Reactance theory (see Brehm's chapter in this volume) predicts that unfavorable cognitive reactions will occur in persuasive situations of a coercive nature. Brock's commodity analysis (see his chapter in this volume) predicts favorability of cognitive reaction to persuasive information to be a decreasing function of the perceived availability of the information. Functional analyses of attitude change (Katz, 1960; Sarnoff, 1960; see also Baron's chapter in this volume) provide more complex principles that might be used to predict cognitive reactions to persuasion given knowledge about the motivational basis of existing attitude structures.

In light of these observations, it would appear fruitful to approach the study of persuasive communication effectiveness with a combination of cognitive process theory and learning theory. The effects of independent variable manipulations in persuasion situations, such as credibility, organization of arguments, communication medium, etc., could be studied simultaneously in terms of their effects on the content of cognitive responses to persuasion and on learning of persuasive information. Existing data, noted at the outset of this chapter, suggest that most of the traditional independent variables of persuasion do not significantly affect retention of persuasive information. (Their effects on attitude presumably are mediated strictly through their effects on cognitive responses to persuasion.) The absence of retention effects of "traditional" persuasion variables may, however, only reflect the fact that attitude change researchers have been more interested in manipulating variables that affect acceptance of persuasive information than ones that affect attention to and retention of persuasive information. In the combined application of cognitive integration theory and learning theory to persuasion, cognitive integration theory should offer an account of the processes involved in acceptance of persuasion while learning theory should seek to account for persistence of induced changes through learning and retention processes. Neither of these areas of theory, when considered alone, can currently be expected to provide a complete account of the processes by which attitudes are formed or lastingly changed in response to persuasive communications.

\section{References}

Anderson, N. H., \& Hubert, S. Effects of concomitant verbal recall on order effects in personality impression formation. Journal of Verbal Learning and Verbal Behavior. 1963, 2, 379-391.

Brehm, J. W., \& Cohen, A. R. Explorations in cognitive dissonance. New York: Wiley, 1962

Brock, T. C. Communication discrepancy and intent to persuade as determinants of counterargument production. Journal of Experimental Social Psychology, 1967, 3, 296-309.

Campbell, D. T. The generality of a social attitude. Unpublished doctoral dissertation, University of California, Berkeley, 1947.

Cooper, Elaine H., \& Pantle, A. J. The total-time hypothesis in verbal learning. Psychological Bulletin, 1967, 68, 221-234.

Fitzgerald, D., \& Ausubel, D. P. Cognitive versus affective factors in the learning and retention of controversial material. Journal of Educational Psychology, 1963, $54,73-84$.

Greenwald, A. G. An amended learning model of persuasion. Paper read at American Psychological Association, Washington, D. C., September 1967.

Greenwald, A. G., \& Albert, Rosita D. Acceptance and recall of improvised arguments. Journal of Personality and Social Psychology, 1968, 8, 31-34.

Greenwald, A. G., Albert, Rosita D., \& Cullen, Dallas M. Persuasion as a function of communication content learning. Unpublished manuscript, Ohio State Univ., 1968.

Greenwald, A. G., \& Sakumura, J. S. Attitude and selective learning: Where are the phenomena of yesteryear? Journal of Personality and Social Psychology, 1967, 7, 387-397.

Hovland, C. I., Janis, I. L., \& Kelley, H. H. Communication and persuasion. New Haven: Yale University Press, 1953.

Hovland, C. I., Lumsdaine, A. A., \& Sheffield, F. D. Experiments on mass communication. Princeton, N. J.: Princeton University Press, 1949

Insko, C. A. Primacy versus recency in persuasion as a function of the timing of arguments and measures. Journal of Abnormal and Social Psychology, 1964, 69, 381-391.

Insko, C. A. Theories of attitude change. New York: Appleton, 1967.

Janis, I. L., \& Gilmore, J. B. The influence of incentive conditions on the success of role playing in modifying attitudes. Journal of Personality and Social Psychology, $1965,1,17-27$.

Janis, I. L. \& Terwilliger, R. F. An experimental study of psychological resistances to fear-arousing communications. Journal of Abnormal and Social Psychology, $1962,65,403-410$

Jones, E. E., \& Aneshansel, J. The learning and utilization of contravaluant material. Journal of Abnormal and Social Psychology, 1956, 53, 27-33.

Jones, E. E., \& Kohler, R. The effects of plausibility on the learning of controversia statements. Journal of Abnormal and Social Psychology, 1958, 57, 315-320. 